\title{
Design of First Generation of Sunan Kalijaga's High Power UV-LED Fluoerescence Spectroscopy System
}

\author{
Frida Agung Rakhmadi ${ }^{1} *$, Rochan Rifai ${ }^{2}$, Khamidinal ${ }^{3}$ \\ ${ }^{1}$ Physics Department; ${ }^{3}$ Chemical Education Department, Faculty of Science and Technology, UIN Sunan Kalijaga \\ Jl. Marsda Adisucipto No 1 Yogyakarta 55281, Indonesia. Tel. +62-274-540971, Fax. +62-274519739. \\ ${ }^{2}$ PT Madeena Karya Indonesia \\ Email*: frida.rakhmadi@uin-suka.ac.id
}

\begin{abstract}
Research on design of the first generation of Sunan Kalijaga's high power UV-LED fluorescence spectroscopy system has been done. The purpose of this research was to make the first generation of Sunan Kalijaga's high power UV-LED fluorescence spectroscopy system. This research was done in two steps, designing and manufacturing of the system. The result showed that a fluorescence spectroscopy system has been made succesfully using high power UV-LED of waveleght $365 \mathrm{~nm}$ as excitation source and webcam MTech WB100 as sensor. The data acquisition software was successfully created using LabVIEW.
\end{abstract}

Keywords: high power UV-LED, fluorescence, spectroscopy

\section{INTRODUCTION}

Spectroscopy is a method often used in material analysis. The discovery of new compounds, both simple and abstruse, can be quickly determined using spectroscopy (Sastrojamidjojo, 2013). One type of spectroscopy widely used was fluorescence spectroscopy. Fluorescence is an atomic or molecular phenomenon that absorbs certain energy and causes a quantum state transition from low energy to high energy level, then emits light with energy lower than the absorbed energy (Lee et al., 2018). Thus, in the fluorescence phenomenon, the energy released is light with a wavelength greater than the wavelength of the excitatory light.

Fluorescence spectroscopy with its variants has been utilized by researchers in various fields. Ghosh et al. (2018) has published one type of fluorescence spectroscopy, namely Fluorescence Lifetime Correlation Spectroscopy (FLCS) which is a development of Fluorescence Correlation Spectroscopy (FCS). In their publication, Ghosh et al. (2018) has written the FCLS theory and its applications. One of requirements for fluorescence was the presence of high-energy light. Some light sources meet this criteria, including LASER and high power LED. Both are currently available on the market with relatively cheap prices. One of the high power LEDs that are currently being sold was high power UV-LED.

In addition to light source with high energy, fluorescence results must also be captured using camera sensors that have adequate resolution. Currently, M-Tech WB100 webcam camera was also available, which was relatively cheap, but the resolution was quite adequate. The two components, both the high-power UV-LED and the M-Tech WB100 webcam, inspired the creation of the first generation of Sunan Kalijaga's high-power UVLED fluorescence spectroscopy system. In addition to these two components, the LabVIEW software was also encouraging. LabVIEW has advantages in the ease of making data acquisition software and its analysis.

\section{MATERIALS AND METHODS}

The research on design of the first generation of Sunan Kalijaga's high power UV-LED fluorescent spectroscopy system was done in five phases: manufacturing of casing, light-emitting sub-system, sample holder sub-system, sensor sub-system, and image recording and analysis sub-system. The five phases above are described as follows. The casing was made of plywood with a length of $30 \mathrm{~cm}$, width of $30 \mathrm{~cm}$, and height of $55 \mathrm{~cm}$. Casing was divided into two parts, top and bottom, each has a door. The upper casing was served to place the image recording sub-system, while the lower casing was served as a place to hang the lightemitting sub-system and place the sample sub-system.

The light-emitting sub-system was made using 4 high power UV-LED 3 watts with wavelength $365 \mathrm{~nm}$ arranged in series, where each lamp is cooled (heatsink). The use of this wavelength was based on the research of Lee et al. (2018) who successfully proved that UV-LED at a wavelength of $365 \mathrm{~nm}$ was able to generate fluorescence in the sample. Making the light-emitting sub-system begins with the manufacture of wooden brackets, followed by the installation of high-power UVLED on the heatsink, then put it on the bracket. The bracket was equipped with a buffer that can be adjusted 
to the height of the sample so that the intensity of the light on the sample can be adjusted.

After that, a driver was installed and connected to the high-power UV-LED. Because each high-power UVLED required a voltage of 3.2-3.8V, a LED driver QH$10 \mathrm{~W}-\mathrm{NF}$ was chosen where it's output voltages were between 9V-14V. Because 4 high-power UV-LEDs were arranged in series, the choice of the driver is right. In addition, the LED driver was equipped with a switching transformer that can be directly connected to $220 \mathrm{~V}$ AC voltage.

The sample holder sub-system was made by acrylic material with long of $18 \mathrm{~cm}$, wide of $18 \mathrm{~cm}$, and width of $5 \mathrm{~mm}$. The sub-system of sample was made permanent, hung on wood. The sensor sub-system consists of a MTech WB100 webcam and a photocopy lens. The use of a photocopy lens was aimed to enlarge the object, so that the M-Tech WB100 webcam catch is more optimal. The camera and lens were placed on the top case. Image acquisition and spectrum analysis sub-system was created using NI Vision LabVIEW 2014. Making of program using graphical data flow techniques. Making of program code was done in NI Vision LabVIEW 2014 in the block diagram window, while the adjustment of the virtual instrument interface was done in the front panel window.

\section{RESULTS AND DISCUSSION}

The first generation of Sunan Kalijaga's high power UVLED fluorescence spectroscopy system was shown in Figure 1. The software of image acquisition and spectrum analysis are shown in Figure 2.

The working principle of the first generation of Sunan Kalijaga's high power UV-LED fluorescence spectroscopy system begins with connecting the power supply subsystem to a 220-volt AC voltage source. Additionally, the sensor sub-system was connected to a 5-volt DC power supply. When the light-emitting subsystem got a power, the four high-power UV-LEDs will emit light illuminating the sample. When high-power UV-LED light illuminates the sample, the atoms in the sample absorb the light energy from the high-power UVLED. The energy absorption will add the energy of atoms in the sample (Lee et al., 2018). If amount of energy absorbed by the atoms corresponds to the excitation energy of the atoms, the atoms will be excited at a higher level of $S^{\prime} 1$. This excitation state takes place in a very short time in the order of nanoseconds. During this time the atoms will experience vibrational relaxation as shown in Figure 3. Under these conditions some energy will be dissipated as heat (Bioscience, 2002).

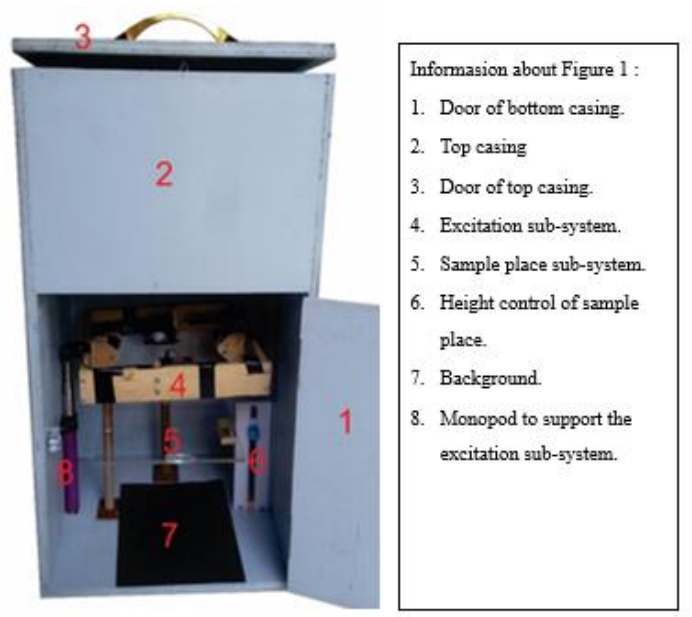

Figure 1. Hardware of the first generation of Sunan Kalijaga's high power UV-LED fluorescence spectroscopy system.

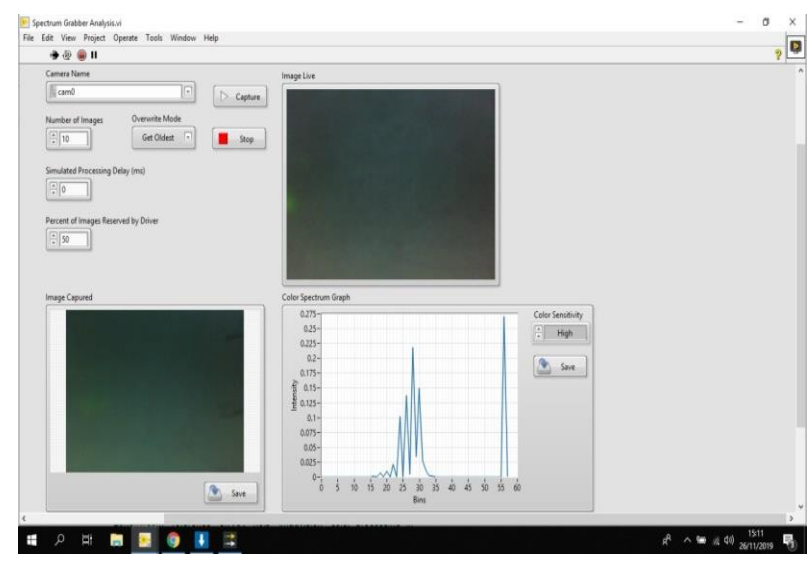

Figure 2. Software of the first generation of Sunan Kalijaga's high power UV-LED fluorescence spectroscopy system.

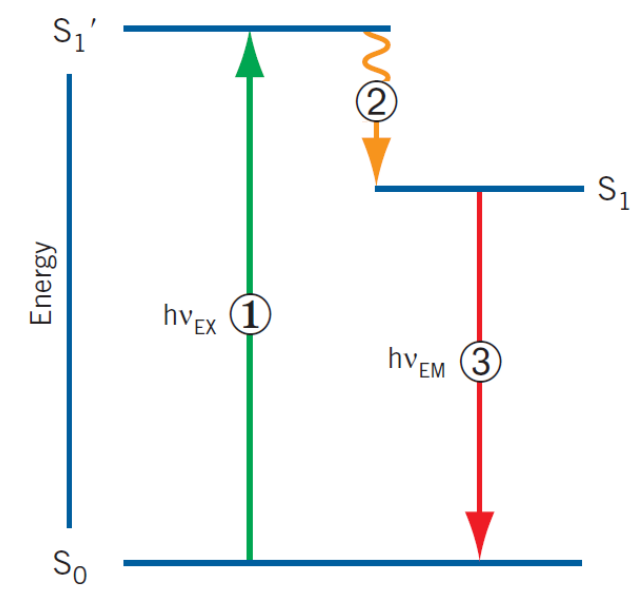

Figure 3. Jablonski Diagram (1) Excitation; Vibrational relaxation; (3) Emission (Bioscience, 2002). 
After reaching the $S^{\prime}{ }_{1}$ state, the atoms will return to the ground state $\left(S_{1}\right)$. When it returns to the ground state, energy is released in the form of light. The released light has a wavelength greater than the wavelength of the excitatory light (Lee et al, 2002). This phenomenon is known as fluorescence. Then, the fluorescence phenomenon was detected using a sensor sub-system (M-Tech WB100 webcam and used photocopy lens). Before detected by the M-Tech WB100 webcam, the image firstly enlarged using a lens. According to Snell's law, the working principle of lens was that the incident ray which hits the front surface of the lens will propagate through the lens and will be refracted (Roychoudhuri, 2008). The M-Tech WB100 webcam will capture fluorescence image and will be displayed in the image acquisition system software as shown in Figure 2. After the image was displayed on the screen, the image will be converted to a spectrum. The spectrum unit in the software was bin, which is a term owned by LabVIEW.

\section{CONCLUSION}

The result showed that a fluorescence spectroscopy system has been made succesfully using high power UVLED of waveleght $365 \mathrm{~nm}$ as excitation source and webcam M-Tech WB100 as sensor. The data acquisition software was successfully created using LabVIEW.

\section{REFERENCES}

Bioscience, Amersham. (2002). Fluorescence Imaging : principles and methods. Amersham Biosciences.

Ghosh, A., Karedla, N., Thiele, J. C., Gregor, I., Enderlein, J. (2018). Fluorescence lifetime correlation spectroscopy: Basics and applications. Methods. www.elsevier.com/locate/ymeth.

Lee, H., Kim, M. S., Lee, W., \& Cho, B. (2018). Sensors and Actuators B: Chemical Determination of the total volatile basic nitrogen ( TVB-N ) content in pork meat using hyperspectral fluorescence imaging. Sensors \& Actuators: $B$. Chemical, 259, 532-539. https://doi.org/10.1016/j.snb.2017.12.102.

Roychoudhuri, Chandrasekhar. 2008. Fundamentals of Photonics. USA: SPIE Press Book

Sastrohamidjojo, Hardjono. (2013). Principles of Spectroscopy. Yogyakarta: UGM Press [Indonesia]. 
THIS PAGE INTENTIONALLY LEFT BLANK 